\title{
Les Entraves au Développement des Villes en Côte d'Ivoire : Cas de la Ville de Sassandra (sud-ouest de la Côte d'Ivoire)
}

\author{
Loba Ilcima Carine, Doctorante \\ Lazare Atta Koffi, Maitre de recherches \\ Armel Frédérique Memel, Maitre-assistant \\ Université Félix Houphouët-Boigny Abidjan, Côte d'Ivoire
}

Doi:10.19044/esj.2019.v15n25p338 URL:http://dx.doi.org/10.19044/esj.2019.v15n25p338

\section{Résumé}

La ville de Sassandra, est située au sud-ouest de la Côte d'Ivoire. Elle constitue le pôle stratégique du redéploiement de production agricole nationale. Cependant, la ville est confrontée à d'énormes difficultés auxquelles s'ajoutent les problèmes spécifiques liés à son site et à la configuration particulière de son territoire. La présente étude se propose d'analyser les entraves au développement de la ville. Pour atteindre cet objectif la méthodologie utilisée est l'analyse documentaire, l'observation directe de terrain et les entretiens avec les acteurs de développement. L'étude révèle que les contraintes au développement sont d'ordres naturels et financiers. Le site de la ville ne facilite pas l'étalement spatial de la ville. La faiblesse des taxes communales, l'instabilité des dotations de l'État et la centralisation de la chaine fiscale sont les principales difficultés économiques de cette ville.

Mots clés : Ville, Développement, Irrégularités topographiques Contraintes budgétaires, Sassandra 


\title{
Impediments to the Development of Cities in Ivory Coast: Case of the City of Sassandra (South West)
}

\author{
Loba Ilcima Carine, Doctorante \\ Lazare Atta Koffi, Maitre de recherches \\ Armel Frédérique Memel, Maitre-assistant \\ Université Félix Houphouët-Boigny Abidjan, Côte d'Ivoire
}

\begin{abstract}
The city of Sassandra, is located in the southwest of Ivory Coast. It constitutes the strategic pole of the redeployment of national agricultural production. However, the city faces tremendous difficulties to which are added the specific problems related to its site and the particular configuration of its territory. The present study proposes to analyze the obstacles to the development of the city. To achieve this objective, the methodology used is documentary analysis, direct field observation and interviews with development actors. The study reveals that the constraints to development are natural and financial. The site of the city does not facilitate the spatial spread of the city. The weakness of communal taxes, the instability of the State's endowments and the centralization of the tax chain are the main economic difficulties of this city.
\end{abstract}

Keywords: City, Development, Topographic, Irregularities, Budget, Constraints, Sassandra

\section{Introduction}

L'un des phénomènes les plus caractéristiques des changements socio-économiques que connaissent aujourd'hui les Etats en développement est l'expansion rapide des zones urbaines. Ainsi en Côte d'Ivoire, l'on assiste à une multiplication des centres urbains. Corrélativement au nombre d'unités urbaines, les populations de ces villes se sont accrues considérablement. De $12 \%$ en 1960 , le taux d'urbanisation est passé successivement à $32 \%$ en 1975 , 39\% en 1988, 43\% en 1998 et à 50,3\% en 2014 (RGPH, 2014).

Cependant, cette croissance a engendré d'énormes difficultés auxquelles doivent faire face les villes, d'autant plus qu'elle se déroule dans un contexte de crise économique.

Ainsi, beaucoup de villes éprouvent des difficultés quand à répondre aux besoins de base des populations. 
Sassandra est une ville moyenne de la Côte d'Ivoire située dans la région du Gboklê. Avec une superficie de 600 hectares et 26608 habitants (source : BNETD, 2010/RGPH, 2014), elle fait partie de la génération des premières villes coloniales ivoiriennes. C'est une ville qui a connu une relative prospérité à l'époque coloniale du fait de son statut de chef-lieu de cercle et d'important port de transit. Erigée en commune de plein exercice au terme de la loi administrative n ${ }^{\circ} 80-1180$ du 17 novembre 1980 et chef-lieu de département de même nom, la ville de Sassandra jouit d'une situation géographique très enviable. Cette cité bénéficie d'un environnement rural très riche. Les données du milieu naturel sont favorables au développement de divers produits agricoles notamment les cultures pérennes et vivrières. En outre sa population cosmopolite est à majorité jeune et active.

Fort de tous ces atouts cette ville du sud forestier devrait être aujourd'hui une ville prospère, l'une des villes les plus importantes du pays. Or que constate-t-on? Lorsqu'on la visite, on observe des équipements et infrastructures déficients et saturés, un cadre de vie dégradé, un environnement perturbé par plusieurs insuffisances. Alors, comment analyser les entraves à sa croissance et son développement ? Ainsi, l'objectif de cette étude est d'analyser les obstacles au développement de la ville de Sassandra.L'hypothèse qui en découle est tt : l'insuffisance des ressources financières est le principal obstacle au développement de la ville.

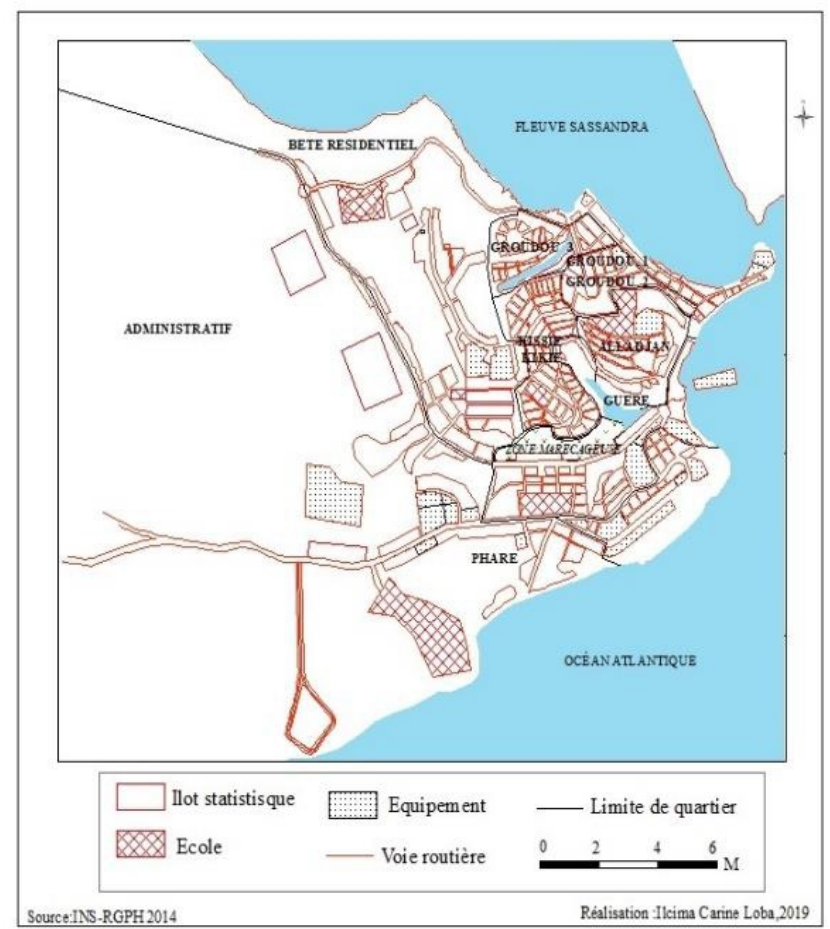

Figure 1 : carte de la ville de Sassandra 


\section{2-Méthodologie}

Pour pouvoir répondre valablement à la question essentielle de cette étude qui s'interroge sur les difficultés de développement de la ville de Sassandra la méthode utilisée est l'analyse documentaire, l'entretien et l'observation directe.

L'analyse documentaire a concerné surtout les programmes triennaux de développement de la municipalité et les différents budgets de la municipalité.

Les entretiens ont été essentiellement faits avec les autorités municipales. L'observation directe a permis à travers des contacts quotidiens d'observer de près l'état du réseau routier, des caniveaux et des équipements socio collectifs.

En ce qui concerne le traitement des données, les logiciels Microsoft Office Word 2013 et Microsoft Office 2007, Excel 2013, le logiciel de SIG ArcGIS 10.2.1 a servi à la réalisation de la carte de présentation de la ville.

\section{3-Résultats}

\section{3-3/Les difficultés de développement de la ville de Sassandra}

\section{3-3-1-Les contraintes naturelles}

Née sur une presqu'ile de la jonction du fleuve et de l'océan, la ville de Sassandra est marquée par une topographie irrégulière. La topographie générale de la ville est tourmentée avec des mamelons de 40 à 80 mètres et des thalwegs marécageux (BNETD, 1998 ; p15) Le site de la ville présente donc une morphologie assez contraignante. Cette contrainte liée à la morphologie accidentée du site de la ville lui confère une organisation irrégulière par la discontinuité du tissu urbain (succession de plateaux et de bas-fonds marécageux). L'habitat est dispersé sur les zones dont le relief permet son implantation. En effet, les zones marécageuses et les versants des mamelons ne peuvent pas être construits sans gros efforts d'aménagement préalables.

\section{3-3-2-Les contraintes économiques}

Le développement urbain est largement dépendant des ressources dont disposent les communes. Les ressources communales se composent de fonds propres provenant des opérateurs économiques locaux, des subventions de l'Etat, des dons et legs. Cependant, l'analyse des différents budgets montre l'instabilité et la faiblesse de celles-ci.

\section{3-3-2-1- Une structure économique encore embryonnaire}

La ville de Sassandra, à l'instar des villes ivoiriennes, est confrontée à des difficultés dans la mobilisation de ressources financières indispensables à son développement. Il s'agit entre autre de la faiblesse des revenus des activités économiques .En effet, celles-ci reposent essentiellement sur le 
primaire et le tertiaire. Les activités industrielles dans la ville de Sassandra sont quasi inexistantes. La figure 2 illustre cette assertion.

Figure 2 : Répartition de la population par branche d'activité en pourcentage

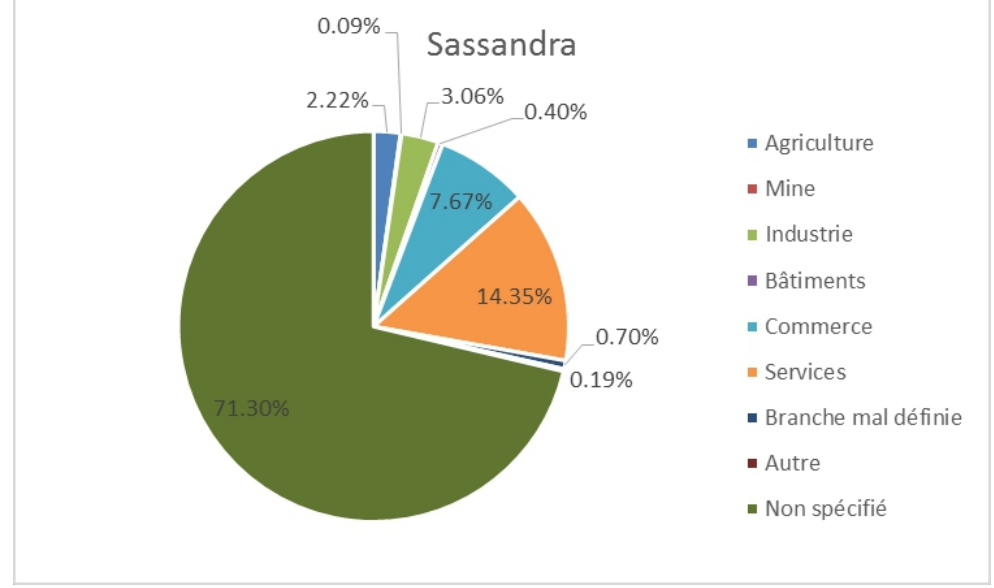

Source : INS-RGPH, 2014

Hormis les non spécifiés, il faut remarquer que ce sont les activités de services qui dominent. Les activités de commerce viennent en seconde position avec un record de $7,67 \%$. On constate ainsi que ce sont les activités relevant du secteur tertiaire qui occupent une place importante. Malheureusement, la ville ne tire pas suffisamment profit de la présence de ces activités. La grande partie des revenus de ces activités ne rentrent pas dans la comptabilité publique. En effet, l'installation désordonnée des acteurs de ce secteur constitue un véritable problème pour les agents de recouvrement de la mairie. Ces derniers ignorent l'effectif réel d'artisans encore moins la localisation de leurs activités.

\section{3-3-2-2- Le faible recouvrement des taxes communales}

La fiscalité locale constitue un jalon essentiel de l'accès aux finances pour les investissements urbains. En effet, une dynamique positive de recette fiscale permet de constituer les garanties nécessaires à la mobilisation de fonds propres indispensables au développement urbain. La fiscalité locale occupe ainsi une place importante dans la structure des budgets communaux. Malheureusement dans la plupart des collectivités et singulièrement à Sassandra, la fiscalité alimente insuffisamment le budget communal. Cela est dû à plusieurs difficultés rencontrées par les autorités communales pour le recouvrement des taxes communales. Le tableau 1 montre l'écart entre les prévisions et les recouvrements de la mairie de Sassandra de 2008 à 2015. 
Tableau 1: Récapitulatif des taxes prévues et recouvrées à Sassandra (en Franc CFA)

\begin{tabular}{|l|l|l|l|l|l|l|l|l|}
\cline { 2 - 8 } \multicolumn{1}{c|}{} & 2008 & 2009 & 2010 & 2011 & 2012 & 2013 & 2014 & 2015 \\
\hline Prévisions & 9927 & 11937 & 16864 & 14026 & 14023 & 15380 & 14008 & 35517 \\
& 0000 & 3700 & 2000 & 7000 & 3000 & 2000 & 9000 & 6000 \\
\hline Recouvre & 7517 & 11775 & 15225 & 10868 & 16979 & 10950 & 23555 & 60879 \\
ment & 3722 & 8779 & 2267 & 8200 & 6737 & 4859 & 5251 & 5620 \\
\hline
\end{tabular}

Source : service financier mairie, 2016

On constate ainsi que dans l'ensemble les prévisions ne sont toujours pas recouvrées. Même si en 2012 et les deux dernières années le recouvrement a largement dépassé les prévisions, les autres années c'est carrément le contraire. L'écart considérable a été enregistré en 2013.Sur les 153802000 Francs CFA prévus c'est seulement 109504859 Francs CFA qui ont été recouvrées. Soit un écart de 44297141 Francs CFA.

\section{3-3-2-3-L'instabilité des dotations de l'Etat}

Depuis la réforme de 1982, les produits des impôts d'Etat perçus par les services publics sont versés aux communes. Ceux-ci sont versés sous forme de dotation globale de fonctionnement (DGF) et de dotation globale d'investissement (DGI). Ces dotations sont instables, elles évoluent en dents de scie. En plus, selon le service financier ces aides sont versées en retard avec des retards de cinq à six mois, souvent même en fin d'année. En effet, non seulement elle arrive en retard, mais les prévisions ne sont toujours pas octroyées; comme en témoigne le tableau ci-dessous. Ce retard entrave considérablement la réalisation de la majorité des projets de développement que la mairie s'est assignée.

Tableau 2: Récapitulatif des Aides de l'Etat prévues et octroyées au Titre I et au Titre II

\begin{tabular}{|l|l|l|l|l|}
\hline \multirow{2}{*}{ Années } & \multicolumn{3}{|l|}{ Titre I (fonctionnent) } & \multicolumn{2}{l|}{ Titre II (investissement) } \\
\cline { 2 - 5 } & Prévisions & Octroyées & Prévisions & Octroyées \\
\hline 2008 & 46000000 & 46000000 & 25000000 & 0 \\
\hline 2009 & 46000000 & 46000000 & 114000000 & 55999460 \\
\hline 2010 & 46000000 & 46000000 & 54000000 & 27181850 \\
\hline 2011 & 46000000 & 44297727 & 30000000 & 30000000 \\
\hline 2012 & 46000000 & 44742332 & 30000000 & 30000000 \\
\hline 2013 & 44298000 & 44297727 & 30000000 & 30000000 \\
\hline 2014 & 41138000 & 41137196 & 37708000 & 37708590 \\
\hline 2015 & 41138000 & 41137196 & 37708000 & 31076783 \\
\hline
\end{tabular}

Source : Service financier mairie ,2016

On constate qu'au titre I (fonctionnement), hormis les trois premières années où les prévisions ont été octroyées, les cinq dernières années c'est le contraire .Cependant l'écart tourne autour d'un million en 2010 et 2011.Pour les autres années, on a moins d'un million comme différence. Au titre II (investissement), les prévisions ont été octroyées entre 2011 et 2013.Concernant les autres années, on a des écarts considérables entre les sommes prévues et celles reçues. 


\section{3-3-2-4-Un budget communal dédié au fonctionnement au détriment des investissements}

Un autre frein au développement dans les communes ivoiriennes en générale et dans celle de Sassandra en particulier, reste et demeure la part du budget de fonctionnement qui est supérieur au budget d'investissement (Figure 3) et ce, au détriment des dispositions de la loi qui prévoit au moins $55 \%$ du budget communal pour les investissements de la commune contre $45 \%$ de ce dernier pour le fonctionnement de la mairie.

Figure 3 : Évolution des budgets d'investissement (Titre II) et de fonctionnement (Titre I) de 2008 à 2015

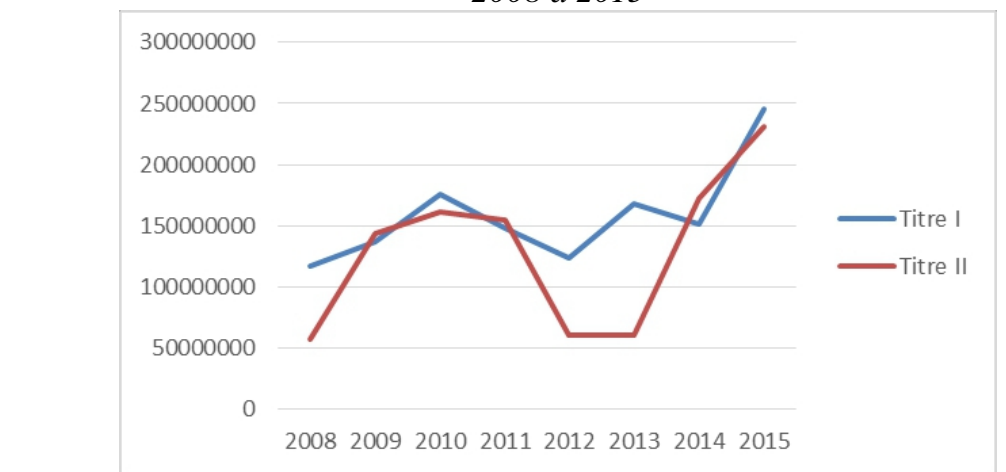

Source : Services financiers des mairies, 2016/Direction des collectivités et du développement local

L'analyse des données secondaires montre un déséquilibre entre les budgets d'investissements et de fonctionnements. En effet, ces données révèlent que le budget de fonctionnement de la commune de Sassandra représente 55\% du budget total de la commune Tandis que celui des investissements représente que $45 \%$. Ce déséquilibre au profit du fonctionnement à un impact négatif sur la mise ne place des projets de développements. Cela se matérialise par un sous équipement notoire en matière $d$ 'infrastructures de drainage, d'assainissement et de voire. L'insalubrité grandissante caractérise le cadre de vie des populations urbaines.

\section{3-3-2-5 Des dispositions institutionnelles contraignantes}

- L'unicité des caisses de l'Etat

En Côte d'Ivoire, le principe de l'unité budgétaire est réaffirmé par un cadre juridique. Il vise à donner un compte unique à toutes les opérations de l'Etat. Toutes les recettes et toutes les dépenses doivent être recrées à ce compte unique qui est le budget de l'Etat. L'objectif est de tendre un peu plus vers la transparence des comptes de l'Etat. De cette façon, toutes les dépenses sont transcrites dans un document unique qui obtient l'autorisation du parlement. La gestion des Caisses est entièrement prise en charge par l'Etat à travers ses services déconcentrés. Dans la pratique, les services des impôts sont chargés du recensement, de l'évaluation de l'assiette et le Trésor du recouvrement. 
C'est également l'Etat qui fixe les taux des différents impôts locaux. L'Etat perçoit les impôts fonciers et taxes, puis reverse une partie aux communes : $40 \%$ des impôts fonciers et $70 \%$ des patentes et licences. L'Etat est donc le receveur unique et définit la part du produit d'impôt collecté qui revient à la commune. Tous les fonds sont mis en commun et gérés par le trésor public. Toute cette procédure empêche les municipalités d'accéder en temps voulu aux fonds qui leur sont affectés. Elles ont parfois du mal à honorer les engagements contractés auprès de leurs différents concessionnaires. Ce qui cause des désagréments car les fournisseurs éprouvent des difficultés à se faire payer après la réalisation des travaux. Ainsi, le pouvoir de décision des collectivités locales en matière financière est très limité.

- Des transferts de compétences limités et sans mesure d'accompagnement

La loi n 85-582 du 29 juillet et la loi n 2003-208 du 07 juillet 2003 portant transfert et répartition des compétences de l'Etat aux collectivités restent explicites à cet égard Cependant dans la pratique des ministères semblent peu disposés à rendre ce transfert effectif. Ainsi au titre des compétences transférées dans les différents domaines plusieurs insuffisances sont à noter. Dans le domaine de l'aménagement et de la planification du développement, on relève l'existence de conflits opposant les communes aux départements dans la mise en œuvre des plans et programmes de développement sur les parties communes de leurs territoires respectifs. En effet, les lacunes en ce qui concerne la définition du territoire communal tel le classement de la voirie et le statut foncier des périphéries urbaines obligent les municipalités à l'impuissance. Aussi, note-t-on l'absence de coordination en matière de planification, de programmation et de mise en œuvre des actions de développement entre communes et départements sur le territoire où ceux-ci se trouvent.

Les compétences transférées aux municipalités requièrent de l'Etat des mesures d'accompagnement en matière de ressources financières, matérielles et humaines. En effet, les ressources financières nécessaires pour assurer les charges au niveau local sont insuffisantes. De nombreuses localités ne peuvent pas disposer de ressources propres satisfaisantes, eu égard au faible niveau d'activités menées et surtout à la difficile définition ou application d'une fiscalité locale concrète. Il ressort que le niveau de prise en charge est décevant. Malgré l'évolution constante de son enveloppe, le montant alloué aux municipalités par l'Etat n'est pas toujours à la hauteur des besoins réels de prise en charge des compétences transférées. En effet, le mode d'évaluation et de compensation retenu par le législateur a eu pour effet de creuser l'écart entre les charges transférées et les ressources versées par l'Etat. 


\section{4- Discussion}

Cette étude a révélé que plusieurs facteurs entravent le développement de la ville de Sassandra et ce en dépit des atouts dont elle dispose.

Dans le même sens A. Loba (2009, p 152) affirme que la communalisation a permis aux villes de disposer de ressources propres en vue de prendre en charge leur développement .Hélas, ces ressources semblent être en nette évolution mais demeurent insuffisantes vue les charges des communes. Ces résultats sont identiques à ceux de F .Memel (2010, p 312-313).

Ce dernier affirme que le faible niveau de développement de la ville de Dabou est imputable à la faible mobilisation des ressources. Le contexte économique étroit de la commune est à la base de cette faiblesse. Aussi toujours selon cet auteur, les dispositions institutionnelles sont contraignantes .En effet, l'Etat dispose encore de l'essentiel du pouvoir fiscal. La main mise de l'Etat dans la gestion locale est à un certain niveau un frein à la mobilisation optimum des finances locales et au développement. Et pourtant l'assainissement financier est un préalable pour restaurer la confiance des populations. De même P. Konan (2010, p 279 ) affirme que l'un des problèmes d'ordre financier le plus récurrent résulte de la mauvaise application des textes régissant la décentralisation.Il est prévu dans les textes de loi que les transferts de compétences par l'État s'accompagnent d'un transfert concomitant aux communes de moyens et de ressources nécessaires à l'exercice normal de ces compétences .Mais ce principe n'est pas respecté dans la pratique. Par exemple, les dépenses de la police municipale relèvent du budget de l'Etat, selon la loi portant organisation des communes. L'article 78 nouveau de cette loi précise que les communes peuvent être appelées à y participer dans la mesure de leurs possibilités financières. Or rarement, le financement de l'État est mis à la disposition des communes pour les dépenses en matière de sécurité, si bien que le coût de la police municipale est supporté uniquement par les communes quels que soient leurs moyens.

\section{Conclusion}

La ville de Sassandra regorge un riche potentiel naturel et humain indispensable pour booster son développement économique et social. Mais cette ville connait un retard en matière de développement. Ce paradoxe s'explique par les nombreuses difficultés rencontrées par les autorités municipales dans l'exercice de leurs fonctions. En effet, les différentes procédures et systèmes mis en place par l'Etat central pour réguler la gestion des autorités locales constituent un handicap quant à la mobilisation des ressources et partant de l'aménagement et l'espace communal. Ce contrôle se manifeste entre autres à travers l'unicité des caisses de l'Etat, la complexité du système fiscal et l'instabilité des dotations de l'État. 


\section{References:}

1. BNETD, 1998.Projet de développement des communes côtières, dossier de préparation du projet, Annexe 2, Abidjan, 249p.

2. BOSSON J, 2012.Contrastes de développement dans l'ancienne boucle du cacao en Côte d'Ivoire : les cas des départements d'Adzopé et de Bongouanou, Thèse de Géographie, Université de Cocody, IGT, 439 p.

3. DURET E, 2000.Réformes institutionnelles et finances publiques. L'exemple de la décentralisation en Afrique Subsaharienne, Thèse nouveau régime, Université d'Auvergne Clermont Fernand I, vol 1, $429 \mathrm{p}$

4. HAUHOUOT A, 2002.Développement, Aménagement, régionalisation en Côte d'Ivoire, Abidjan EDUCI, 359p

5. KIPRE P, 1985.Villes de Côte d'Ivoire de 1893 à 1940 Tome I, Nouvelles éditions Africaines, 238p

6. KONAN P, 2010. Développement urbain en Côte d'Ivoire : cas de la ville de Dimbokro, Thèse unique de Géographie Université de Cocody Abidjan,336 p

7. LOBA A, 2009.Dynamique de développement des villes côtières, Thèse unique de géographie Abidjan, Université de Cocody, 390p

8. MEMEL F, 2012.Ressources communales et Aménagement urbain en Côte d'Ivoire : cas de la ville de Dabou, Thèse unique de Géographie Abidjan, Université de Cocody, 353p

9. MINISTERE DE LA CONSTRUCTION ET DE L'URBANISME, 2016.PUD, Diagnostic stratégique Sassandra, 109p

10. TAPE S, 2012.Aménagement et gestion des villes du sud-ouest du littoral ivoirien, thèse unique de géographie, Université de Cocody Abidjan, 475p

11. ZOMBO J P, MAI G H W, ALOKO-N'GUESSAN J., 2018 « Apport des collectivités dans le développement local : Cas de la mairie de Guiglo (Ouest de la Cote d'Ivoire) », In Villes et Développement en Afrique Subsaharienne, Mélanges en l'honneur du Professeur Emérite Benoît D. N'BESSA, Tome 2, Université d'Abomey-Calavi Cotonou, Benin, pp $216-233$ 